\title{
BIOMETRY OF PERMANENT OCCLUSION DENTAL ARCHES - COMPARISON ALGORITHM FOR REAL AND DESIGN INDICATORS
}

\section{Victoria Borodina', Dmitry Domenyuk2, Lyudmila Weisheim ${ }^{3}$, Sergei Dmitrienko ${ }^{1}$}

\author{
Pyatigorsk Medical-Pharmaceutical Institute, Pyatigorsk \\ Stavropol state medical university, Stavropol \\ Volgograd state medical university, Volgograd
}

The measuring accuracy of the dental arches linear parameters plays a crucial role in diag-nosing abnormalities and deformations in the dentoalveolar system. The major research method up to date has been the biometric study of jaw stone models $[1,5]$. Measuring implies a compara-tive evaluation of the dental arches dimensions against the dimensions of the teeth that constitute the dentition [6]. There have been correspondence indices proposed, the best known of them being the data proposed by Pont, Linder-Harth, Korkhaus, Howe, and by other researchers. At the same time, their advantages and disadvantages have been revealed, with the most rational approaches identified in view of the gnathic and dental types of the dental arches $[2,4]$. Correlation has been identified between the dental arch size and the craniofacial parameters, as well as their role in selecting orthodontic treatment [3]. Researchers and orthodontists typically take teeth and dental arch measurements manually using various modifications of dental calipers. These methods are time- and effort-consuming, whereas potential errors cannot be ruled out. The available literature offered us no data that would allow comparing the actual values with the de-sign parameters, which was the aim of the study.

Arm. To develop an algorithm for comparing the actual and the design indicators at bio-metric examination of the permanent occlusion dental arches.

MATERIALS AND METHODS: The study involved 79 persons aged 20 to 25 , with no signs of dental pathology and with a full set of permanent teeth. All the participants had their teeth imprints taken on elastic stuff to further transfer the imprints on die-stone models. The biometric measurement was performed with calipers (accuracy $-0.01 \mathrm{~mm}$ ). In the anterior section, the mesial-distal width of the canines and incisors was measured. The length of the entire dental arch was the width sum of the crowns of the 14 teeth that constitute the dentition. Transversally, the dental arch width between the canines was measured at two points - between the canines cusps and between the canines distal surfaces at the vestibular surface. The width between the second molars was measured between the points located on the peaks of the vestibular distal odontomers cusps. Specific attention was paid to the diagonal dimensions of the dental arches, while the reference point was interincisal point, from which the distance to the canines and second molars location level was measured.

RESULTS AND DISCUSSION. The measurement revealed people with permanent orthognathic occlusion had their upper jaw dental arch anterior section length equal to $46.78 \pm 0.82 \mathrm{~mm}$, while at the lower jaw the similar measure produced $36.58 \pm 0.67 \mathrm{~mm}$. The ratio of these parameters, which was $1.28 \pm 0.06$, was used as a criterion for comparing the length of the jaws anterior part. The upper jaw dental arch length was $114.5 \mathrm{~mm}$, at the lower jaw $-108.34 \mathrm{~mm}$, whereas the value of the compliance coefficient of $1.06 \pm 0.01$ was recommended to be used when comparing the obtained actual values. The values of the ratio of the dental arch length to the length of the anterior part of the arch (upper jaw -2.45 , lower jaw -2.96 ) were used to develop the corre-spondence algorithm for the said parameters. The width of the dental arch anterior part, meas-ured between the canine tearing cusps, was $39.4 \pm 0.26 \mathrm{~mm}$ and $31.76 \pm 0.19 \mathrm{~mm}$ for the upper and lower jaws, respectively. At the same time, the transversal dimensions between the canines distal surfaces were $2 \mathrm{~mm}$ larger $(41.32 \pm 0.29 \mathrm{~mm}$ and $33.79 \pm 0.17 \mathrm{~mm}$, respectively). The ratio of the dental arch length to the dental arch diagonal dimensions, in particular to the frontal-distal diagonal, was 2.12 , and the difference between the design and the actual values did not exceed $2 \mathrm{~mm}$ measuring the linear parameters of dental arches and individual teeth allowed developing an algorithm for comparing the actual and the design indicators at permanent occlusion dental arches biometry. The algorithm included a sequence of steps. First, the length of the anterior part of the upper jaw dental arch was measured. Its ratio to the 1.28 coefficient value showed the estimated length of the anterior section of the lower jaw dental arch. After measuring the actual value, the indicators were compared. If the design and the actual values did not match, the measurement was done over. The indices deviation can be used for diagnosing anomalies in the anterior teeth size. Second, the length of the upper jaw 
dental arch was identified. Its ratio to the coefficient of 1.06 showed the estimated length of the lower jaw dental arch. The outcomes were interpreted similarly to the length of the dental arch anterior section. Third, the ratio of the dental arch length to the dental arch diagonal dimensions was estimated using the coefficient of 2.12. Normally, the difference in the design and in the actual values should not exceed $2 \mathrm{~mm}$.

Conclusion. The proposed algorithm for comparing the design and the actual indicators at biometrics of permanent occlusion dental arches offers a way to obtain accurate data concerning the dental arches dimensions for both jaws, as well as to reveal dentoalveolar pathologies. The algorithm included a series of steps to measure the length of the dental arches anterior section, the entire dental arch length, the arch width at the canine and the molar region, and to determine diagonal dimensions employing the proposed compliance coefficients.

\section{REFERENCES}

1. Domenyuk, D.A. Correlation of dental arch major linear parameters and odontometric indices given physiological occlusion of permanent teeth in various face types / D.A. Domenyuk, E.G. Vedeshina, S.V. Dmitrienko // Archiv EuroMedica. - 2016. - T. 6. № 2. - C. 18-22.

2. Domenyuk, D.A. Mistakes in Pont (Linder-Harth) method used for diagnosing abnormal dental arches in transversal plane / D.A. Domenyuk, E.G. Vedeshina, S.V. Dmitrienko // Archiv EuroMedica. - 2016. - T. 6. - № 2. - C. 23-26.

3. Domenyuk D.A., Shkarin V.V., Porfiriadis M.P., Dmitrienko D.S., DMitrienko S.V. Classification of facial types in view of gnathology // Archiv EuroMedica, 2017. - T.7. - № 1. - P. 8

4. Domenyuk, D.A. Modern classification of dental arches / D.A. Domenyuk, S.V. Dmitrienko // Archiv EuroMedica, 2014. - Vol. 4. - № 2. - P. 14-16.

5. ShKarin V.V., Domenyuk D.A., Porfiriadis M.P., DMitrienko D.S., DMitrienko S.V. Mathematical and graphics simulation for individual shape of maxillary dental arch // Archiv Euro-Medica, 2017. - T. 7. - № 1. - C. 60-65.

6. Shkarin V.V., Porfiriadis M.P., Domenyuk D.A., DMitrienko D.S., Dmitrienko S.V. Setting reference points for key teeth location in case of abnormal dental arch shape //Archiv EuroMedica. - 2017. - V.7. - № 2. - C. 111-117. 